\title{
Dual inhibition of thrombin and activated factor $X$ attenuates disseminated intravascular coagulation and protects organ function in a baboon model of severe Gram-negative sepsis
}

\author{
Herbert Schöchl ${ }^{1,2^{*}+}$ (1), Martijn van Griensven ${ }^{3+}$, Stefan Heitmeier ${ }^{4}$, Volker Laux ${ }^{4}$, Ulrike Kipman ${ }^{5}$, Jan Roodt ${ }^{6}$,
} Soheyl Bahrami ${ }^{1}$ and Heinz Redl ${ }^{1}$

\begin{abstract}
Background: Inhibition of procoagulant pathways may improve outcome in sepsis. We examined whether a dual short-acting thrombin (factor II) and factor X (FX)a inhibitor (SATI) ameliorates sepsis-induced disseminated intravascular coagulation (DIC) and is organ-protective.

Methods: Escherichia coli were infused for $2 \mathrm{~h}$ in 22 anesthetized baboons. The control $(\mathrm{CO})$ group $(\mathrm{n}=8)$ received sterile isotonic solution only. In the treatment groups, SATI was administered starting 15 minutes after the end of the bacterial exposure. In the low-dose group (LD-SATI, $n=8$ ), SATI was infused with $75 \mu \mathrm{g} / \mathrm{kg} / \mathrm{h}$ for the first hour, followed by $23 \mu \mathrm{g} / \mathrm{kg} / \mathrm{h}$ until the end of the study. In the high-dose SATI group (HD-SATI, n=6), $225 \mu \mathrm{g} / \mathrm{kg} / \mathrm{h}$ was administered for the first hour followed by continuous infusion of $69 \mu \mathrm{g} / \mathrm{kg} / \mathrm{h}$ until termination of the study.

Results: Sepsis-induced DIC was attenuated, as reflected by lower peak thrombin-antithrombin complexes (threefold) and D-dimer levels (twofold) in both SATI groups compared to the CO. This coincided with strongly improved cell/organ protection assessed by decreased levels of lactate dehydrogenase (threefold), creatinine (twofold), aspartate aminotransferase (threefold), and amylase (twofold) compared to the CO group. Anuria, which started at $8 \mathrm{~h}$ in the CO group, was prevented in both SATI groups. Peak interleukin- 6 release at $12 \mathrm{~h}$ was prevented in the treatment groups. In both SATI groups, fewer catecholamines were necessary and no bleeding complications were observed.
\end{abstract}

Conclusions: Dual inhibition of thrombin and FXa preserved activation of coagulation, protected organ function and ameliorated inflammation in severe Gram-negative sepsis in baboons. SATI could be a novel therapeutic agent against sepsis-induced DIC.

Keywords: DIC, Inflammation, MOF, Short-acting coagulation factor II/Xa inhibitor, SATI

\footnotetext{
*Correspondence: herbert.schoechl@auva.at

${ }^{\dagger}$ Equal contributors

${ }^{1}$ Ludwig Boltzmann Institute for Experimental and Clinical Traumatology, AUVA Research Centre, Vienna, Austria

${ }^{2}$ Department of Anesthesiology and Intensive Care Medicine, AUVA Trauma

Centre Salzburg, Academic Teaching Hospital of the Paracelsus Medical

University, Dr. Franz Rehrl Platz 5, 5020 Salzburg, Austria

Full list of author information is available at the end of the article
}

C The Author(s). 2017 Open Access This article is distributed under the terms of the Creative Commons Attribution 4.0 International License (http://creativecommons.org/licenses/by/4.0/), which permits unrestricted use, distribution, and reproduction in any medium, provided you give appropriate credit to the original author(s) and the source, provide a link to the Creative Commons license, and indicate if changes were made. The Creative Commons Public Domain Dedication waiver (http://creativecommons.org/publicdomain/zero/1.0/) applies to the data made available in this article, unless otherwise stated. 


\section{Background}

Severe sepsis and septic shock remain a prominent cause of mortality in hospital, exceeding 30\% [1, 2]. Bacterial invasion not only prompts a potent systemic inflammatory response, but it also results in compelling upregulation of the coagulation system [3-7]. Thus, the majority of patients with severe sepsis suffer from coagulation disorders ranging from minor changes in activation markers to full-blown disseminated intravascular coagulation (DIC) $[7,8]$.

Experimental studies demonstrate that tissue factor (TF) expression, in particular on monocytes and endothelial cells play a key role in the pathogenesis of DIC [7]. TF complexes with factor VII (FVII) and subsequently activates factor X (FX) and factor IX (FIX) with a strong downstream effect on thrombin generation [9]. This procoagulant stimulus in alliance with sustained depression of fibrinolytic pathways results in disseminated intravascular formation of microthrombi [5]. Widespread fibrin deposition in the microcirculation of various organs is closely linked to the development of multiple organ failure and has been identified as an important contributor of morbidity and mortality $[5,10]$. For example, Dhainaut et al. reported that mortality increased from $27 \%$ in patients with sepsis without DIC to $43 \%$ in those with accompanying DIC [11].

Restoration of anticoagulant pathways by administration of antithrombin (AT) III, recombinant activated protein $\mathrm{C}$ (APC) and recombinant tissue factor pathway inhibitor (TFPI) in patients with sepsis without DIC has not resulted in survival benefits in randomized controlled trials [12-16]. In contrast, ATIII and recombinant APC in patients with overt DIC showed promising results towards improved outcome [17-19].

Direct thrombin inhibitors such as lepirudin and the predominant factor Xa inhibitor Danaparoid have been investigated in endotoxin models. Both substances are sufficient to hinder activation of coagulation [20,21]. Until now, no studies investigated a combined thrombin and FXa inhibitor in severe sepsis. Dual inhibition of thrombin and FXa in part mimics the natural anticoagulant ATIII, which effectively inhibits both enzymes [22]. While ATIII binds thrombin in a 1:1 ratio, direct thrombin and FXa inhibitors block the active side of the corresponding coagulation factors and are not dependent on sufficient levels of cofactors such as heparin [23].

In the current study, we examined the efficacy and safety of a new potent, short-acting, reversible and selective combined FIIa and FXa inhibitor (short-acting thrombosis inhibitor (SATI)) in an established experimental model of severe Escherichia coli sepsis in baboons [24]. The hypothesis of the current investigation was that the administration of SATI in severe sepsis will attenuate activation of coagulation (with consecutive microthrombi formation), diminish DIC and preserve organ function.

\section{Methods}

The experimental protocol was approved by the Institutional Animal Care use Committee at Free State University Bloemfontein, South Africa (number 03/2010). All experiments were performed under the conditions described in the Guide for the Care and Use of Laboratory Animals as defined by the National Institutes of Health.

Twenty-two healthy male Chacma baboons of the strain Papio ursinus, with a median weight of 18.1 (16.5-22.3) $\mathrm{kg}$ and aged between 3 and 10 years were included in the current study. The animals were quarantined for 3 months prior to the study. The primates were fasted overnight before experiments, with free access to water.

\section{Premedication, anesthesia and instrumentation of the animals}

Premedication of the baboons was performed by intramuscular injection of $6-8 \mathrm{mg} / \mathrm{kg}$ body weight (BW) ketamine hydrochloride (Ketalar ${ }^{\oplus}$, Pfizer, Vienna, Austria). After placing the animal in the supine position, the right cubital vein was cannulated and anesthesia was induced using $5 \mathrm{mg} / \mathrm{kg}$ BW sodium pentobarbital (Sandoz $\mathrm{GmbH}$, Kundl, Austria) and maintained by continuous infusion of $0.8 \mathrm{mg} / \mathrm{kg} / \mathrm{h}$ sodium pentobarbital, $0.8 \mu \mathrm{g} / \mathrm{kg} / \mathrm{h}$, sufentanil (Janssen, Vienna, Austria), and $1 \mathrm{mg} / \mathrm{kg} / \mathrm{h}$ rocuronium (Organon, Oss, Netherlands).

Following endotracheal intubation the animals were ventilated in volume-controlled mode using a tidal volume of $6-8 \mathrm{ml} / \mathrm{kg}$ and a respiratory rate of $18-20$ breaths/ minute in order to maintain an arterial pressure of $\mathrm{CO}_{2}$ at 35-45 mmHg (Evita 2, Dräger, Lübeck, Germany). The fraction of inspired oxygen was set at 0.30 and adjusted if necessary according to blood gas analyses. Body temperature was kept at $37^{\circ} \mathrm{C}$.

Three catheters were placed using the Seldinger technique: (1) for blood pressure monitoring and blood sampling a catheter was inserted into the right femoral artery; (2) the cephalic vein was used for fluid therapy and administration of SATI; and (3) a Swan-Ganz catheter (Edwards Lifesciences, Irvine, CA, USA) was inserted via the femoral vein for measurement of central venous pressure (CVP), pulmonary arterial pressure and, pulmonary artery occlusion pressure (PAOP), and hemodynamic variables such as cardiac index $(\mathrm{CI})$, systemic vascular resistance index (SVRI) and pulmonary vascular resistance index (PVRI). A transurethral catheter was placed to quantify urine output at baseline, and at 2-h intervals for $8 \mathrm{~h}$, followed by 4-h intervals for $24 \mathrm{~h}$ ).

\section{Experimental design}

After a stabilization period, $5 \times 10^{6}$ colony-forming units (CFU)/kg E. coli O111:K58(B4):H-(ATCC 33780) were infused under continuous stirring over a $2-\mathrm{h}$ period via a motor-pump as previously described [25] (Fig. 1). 


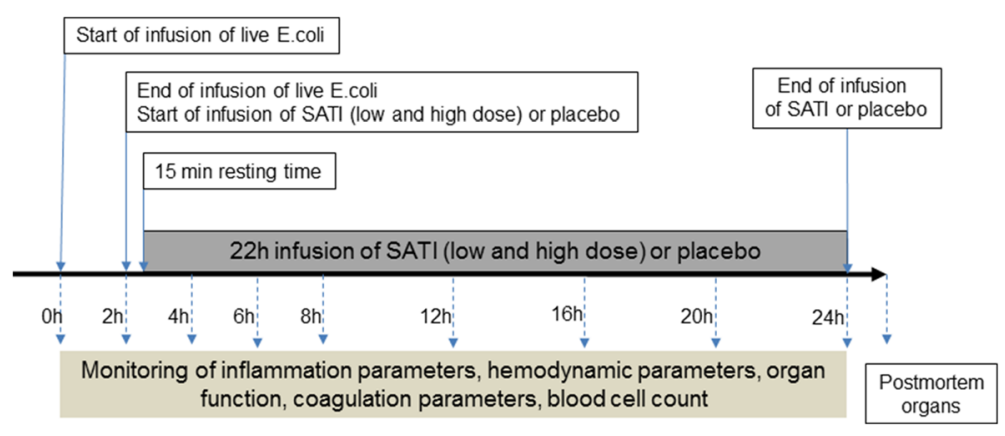

Fig. 1 Study protocol. SATI short-acting thrombosis inhibitor

Ringer's solution (Fresenius-Kabi GmBH, Bad Homburg, Germany) of at least $5 \mathrm{ml} / \mathrm{kg} / \mathrm{h}$ was infused to maintain mean arterial blood pressure (MAP) at $>70 \mathrm{mmHg}$ and $\mathrm{CVP}$ and PAOP at $>5 \mathrm{mmHg}$. If these target values were not established, higher rates of Ringer's solution or noradrenalin (or if necessary adrenalin) were administered. Antibiotics were not used.

To investigate the optimal dose for efficacy and safety, two treatment regimens were evaluated: for the lower dose a plasma concentration was selected that led to a prothrombin time (PT) prolongation of 1.4 in healthy animals. This concentration was chosen, because it resulted in a significant reduction in thrombus weight of about $50 \%$ in an arteriovenous shunt thrombosis model in rats, which has previously been used for predictions of concentration in the setting of acute thrombosis. As expected, a threefold higher concentration led to approximately $80 \%$ reduction in thrombus weight, but it did not lead to a significant increase in bleeding time. Thus, these two doses were chosen to investigate whether the lower dose is as efficacious as in acute arterial thrombosis scenarios and whether the higher dose would have even more efficacy with an acceptable bleeding profile.

At 15 minutes after completion of the bacterial infusion the animals were randomly assigned to one of three groups. The control $(\mathrm{CO})$ group $(\mathrm{n}=8)$ received a crystalloid solution as vehicle (Ionosteril, Fresenius-Kabi GmBH, Bad Homburg, Germany). The treatment groups received either low-dose SATI (LD-SATI) or high-dose SATI (HD-SATI). The LD-SATI group $(\mathrm{n}=8)$ started with $75 \mu \mathrm{g} / \mathrm{kg} / \mathrm{h}$ for the first hour followed by $23 \mu \mathrm{g} / \mathrm{kg} / \mathrm{h}$ until the end of the study. In the HD-SATI group $(n=6)$, $225 \mu \mathrm{g} / \mathrm{kg} / \mathrm{h}$ was administered for the first hour followed by continuous infusion of $69 \mu \mathrm{g} / \mathrm{kg} / \mathrm{h}$ until the end of the study. SATI was dissolved in $50 \mathrm{ml}$ of vehicle. At $24 \mathrm{~h}$ after the start of the bacterial infusion, the animals were killed by an intravenous (i.v.) bolus injection of $100 \mathrm{mg} / \mathrm{kg}$ pentobarbital followed by $10 \mathrm{ml}(1 \mathrm{mmol} / \mathrm{ml})$ potassium chloride.

\section{Blood sampling}

Baseline blood samples for blood gas analysis, blood cell count and clinical chemistry analysis were obtained after $1 \mathrm{~h}, 2 \mathrm{~h}$ and then at every $2 \mathrm{~h}$ within the first $8 \mathrm{~h}$, followed by sampling every $4 \mathrm{~h}$ until the end of the experiment (24 h) (Fig. 1). Blood cell counts were determined by a Coulter T890 counter (Coulter Electronics Inc., Hialeah, FL, USA). Coagulation analyses were performed in the blood collected in 3-ml tubes containing $0.3 \mathrm{ml}$ buffered $3.2 \%$ trisodium citrate, giving a volume ratio of $1+9$ (Vacuette; Greiner Bio-One, Linz, Austria). Blood was centrifuged immediately at $2000 \mathrm{~g}$ for 10 minutes to obtain plasma, which was stored at $-80^{\circ} \mathrm{C}$ until analysis.

\section{Coagulation tests}

The coagulation tests were performed according to the manufacturer's instructions: antithrombin (AT) (AssayMax human Antithrombin III ELISA; AssayPro, St. Charles, MO, USA); D-dimer (Asserachrom D-Dimer ELISA, Diagnostica Stago, Taverny, France); fibrinogen (Human Fibrinogen ELISA, ICL, Portland, USA); PAI-1 antigen (Technozym ELISA, Cryopep, France); activated protein C (human activated Protein C ELISA, Wuhan USCNK, Houston, USA); thrombin-antithrombin-complexes (Enzygnost TAT micro, ELISA, Siemens, Marburg, Germany); tissueplasminogen-activator activity (Technozym ELISA t-PA, Technoclone, Vienna, Austria); tissue factor pathway inhibitor (Quantikine human TFPI ELISA, R\&D Systems, Minneapolis, USA), thrombin activated fibrinolysis inhibitor (Zymotest TAFI Antigen; Hyphen, Neuville-sur-Oise, France) and thrombomodulin (Diagnostica Staro, Taverny, France).

\section{Inflammation markers}

Lipopolysaccharide binding protein (LBP), interleukin (IL)-6, and IL-10 were determined using ELISA assays and performed on Immulite 1000 (Siemens Healthcare, Erlangen, Germany). Tumor necrosis factor- $\alpha$ (TNF- $\alpha$ ) was measured by ELISA kits (Bender MedSystems, 
Vienna, Austria) according to the manufacturer's instructions.

\section{Blood gases and chemistry}

Arterial and venous blood gases were measured (Radiometer ABL 330, Copenhagen, Denmark). Renal (urea, creatinine, uric acid), liver (aspartate-Aminotransferase (AST), alanineaminotransferase (ALT) and bilirubin) and pancreas parameters (amylase, lipase) and cell damage parameters (lactate dehydrogenase (LDH) were analyzed using the Sysmex XE-2100 (Sysmex, Kobe, Japan).

\section{Statistical analyses}

To investigate group differences and changes in organ function parameters due to the intervention in an overall analysis, we performed repeated measures analysis of variance (ANOVA) with time as a within-subjects-factor and group as a between-subjects-factor for each parameter. The start of SATI administration was chosen as the reference time point for the comparisons between the $\mathrm{CO}$ and treatment groups. Post hoc analyses were conducted using the Scheffé test. Analysis of differences was performed separately for each time point using the $t$ test for independent samples (Bonferroni-corrected). For categorical variables, the chi square test was used. $P$ values $<0.05$ were considered statistically significant. All statistical calculations were performed using commercially available statistical software (SPSS 23.0) and figures were created using GraphPad Prism 5.03. Data are expressed as mean and standard deviation (SD) or median and interquartile range.

\section{Results}

Of the 22 baboons, 20 survived until the end of the experiment; the two deaths were encountered in the LD-SATI group. Importantly, no severe and/or life-threating bleeding complications (e.g. mucosal bleeding, bleeding from catheter insertion sites or hematuria) were observed either in the CO group or in either of the SATI treatment groups.

\section{Sepsis-related coagulopathy}

The bacterial challenge resulted in strong procoagulant activation indicated by high thrombin-anti-thrombin (TAT) complexes in the $\mathrm{CO}$ group. SATI administration abolished TAT complex formation $(p<0.0001)$ in the treatment groups compared to the $\mathrm{CO}$ group (peak, threefold higher), and significantly attenuated fibrinogen consumption in the HD-SATI group (HD-SATI vs the CO group, $p<0.02$ ). All animals developed severe thrombocytopenia after infusion of E. coli, without any significant differences between the $\mathrm{CO}$ group and the two SATI groups (Fig. 2a-c).

ATIII significantly decreased over time in all groups $(p<0.0001)$, but only LD-SATI preserved ATIII consumption $(p \leq 0.02)$. APC increased significantly until the end of the experiment $(p<0.0001)$. HD-SATI administration resulted in lower APC values compared to the $\mathrm{CO}$ group (time point $8 \mathrm{~h}$ from baseline (TP 8), $p<0.014)$. Soluble Thrombomodulin (sTM) strongly increased over the observation period without significant differences between groups (data not shown). In the CO group, TFPI increased significantly until the end of the experiment $(p<0.0001)$. Animals in both treatment groups had lower TFPI concentrations compared to those in the CO group (both $p<0.0001$ ). In particular, HD-SATI abolished upregulation of TFPI almost completely (Fig. 3a-c).

D-Dimers significantly increased over time in all groups reaching the highest level at TP $20(p<0.0001)$. Significant differences between the CO and LD-SATI groups $(p \leq$ $0.005)$ and the HD-SATI group $(p \leq 0.001)$ were detected.

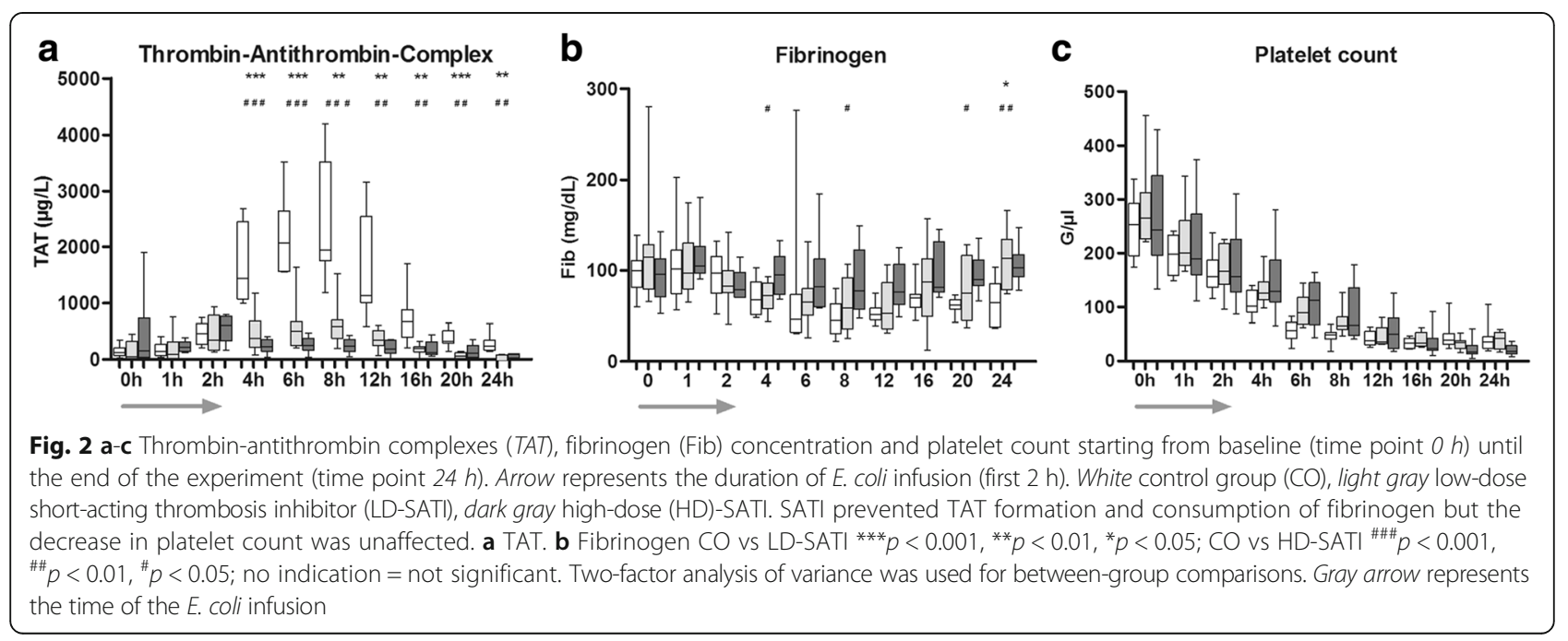




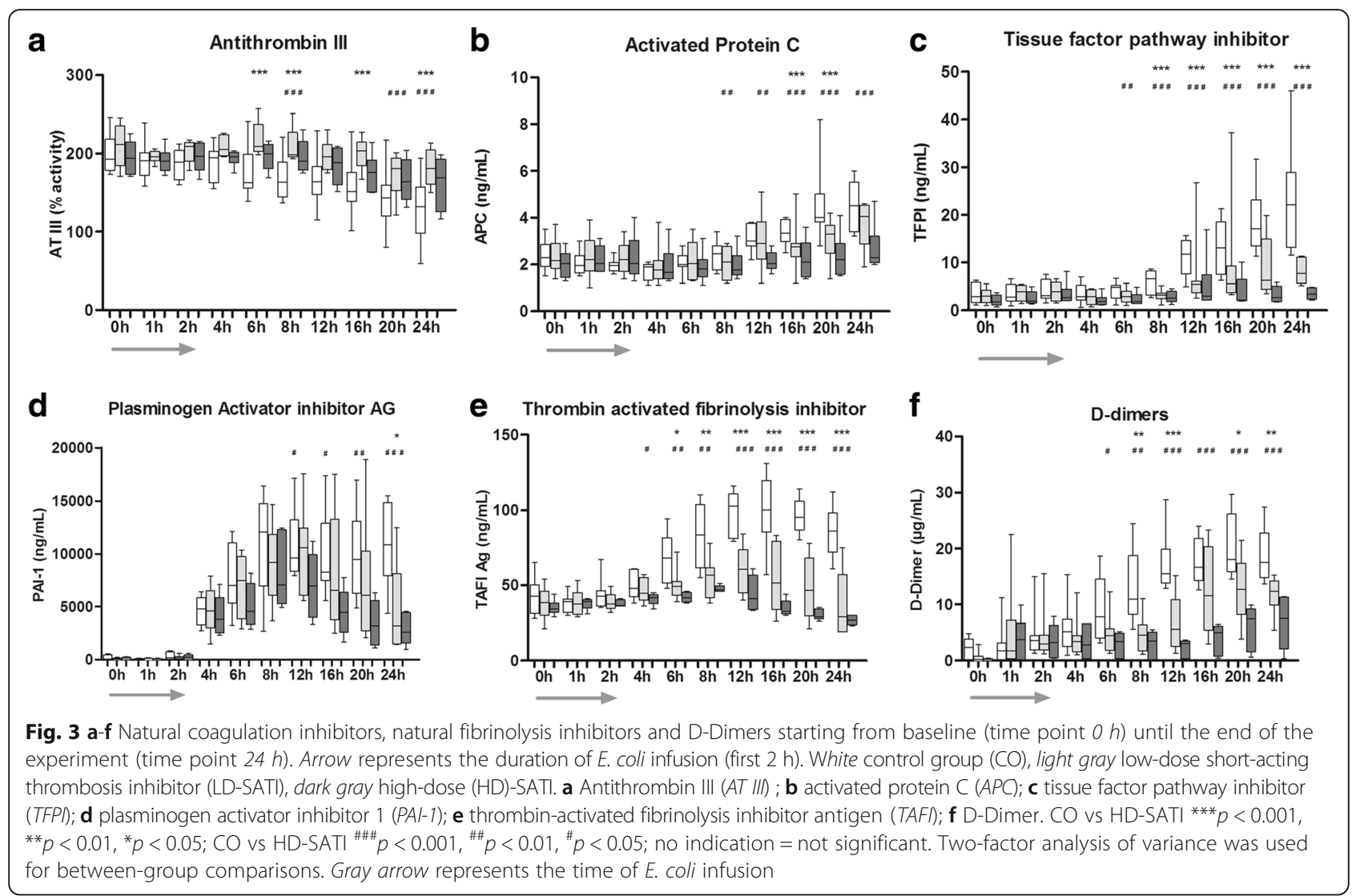

D-dimers at TP 12 were twofold higher in the CO compared to the treatment groups. HD-SATI appeared to be more efficient in attenuating D-Dimer production compared to LD-SATI. Plasminogen activator inhibitor 1 antigen (PAI-1) significantly increased over the observation period in all groups reaching the highest levels at TP 12. HD-SATI treatment resulted in significantly lower levels of PAI-1 compared to control $(p=0.03)$. Thrombinactivated fibrinolysis inhibitor antigen (TAFI) strongly increased in the $\mathrm{CO}$ group reaching the highest level at TP $16(p<0.0001)$. TAFI activation was sufficiently attenuated $(p<0.0001)$ in both SATI groups (Fig. 3d-f).

\section{Sepsis-induced organ damage}

Oxygenation significantly deteriorated over the observation period in all groups as indicated by a steady decrease in the ratio of arterial oxygen partial pressure to fractional inspired oxygen $\left(\mathrm{paO}_{2} / \mathrm{FiO}_{2}\right)$ ratio $(p<0.0001)$ (Fig. 4a) without significant differences between groups.

All animals in the CO group were anuric after TP 8, while urine production did not stop in any of the SATItreated baboons. Both creatinine and urea significantly increased over time in all groups. However, creatinine was twofold higher in the CO group compared to both SATI treatment arms at TP 24 $(p<0.0001)$. Similarly, urea was twofold higher in the CO compared to the HD-SATI group $(p \leq 0.03)$ (Fig. $4 b, c)$.

Treatment with LD-SATI and with HD-SATI lowered circulating AST threefold compared to $\mathrm{CO}$ at $24 \mathrm{~h}(p<$ $0.001)$. Amylase increased significantly in the course of the study $(p<0.005)$; SATI treatment (regardless of the dose) significantly abolished release of amylase $(p<0.05)$ compared to CO. LDH strongly increased over time in all groups $(p<0.0001)$. SATI treatment in both dose groups resulted in threefold lower $\mathrm{LDH}$ release compared to the CO (both $p<0.0001$ ), (Fig. 4 d-f).

\section{Inflammation parameters after $E$. coli administration}

In all groups, bacterial infusion caused a strong increase in circulating inflammatory cytokines. TNF- $\alpha$ and IL-10 peaked, prior to treatment, $2 \mathrm{~h}$ after the start of bacterial infusion without differences among groups. IL-6 significantly changed over time $(p<0.001)$. The highest concentration of IL- 6 was reached $10 \mathrm{~h}$ after termination of the bacterial infusion (TP 12) in the $\mathrm{CO}$ group. In contrast, maximum IL- 6 concentrations were measured at TP 8 in the LD-SATI group and at TP 6 in the HD-SATI group, respectively. SATI treatment in both arms significantly attenuated the magnitude of IL- 6 release compared to $\mathrm{CO}$ (both $p<0.0001$ ) (Fig. 5a-c). 


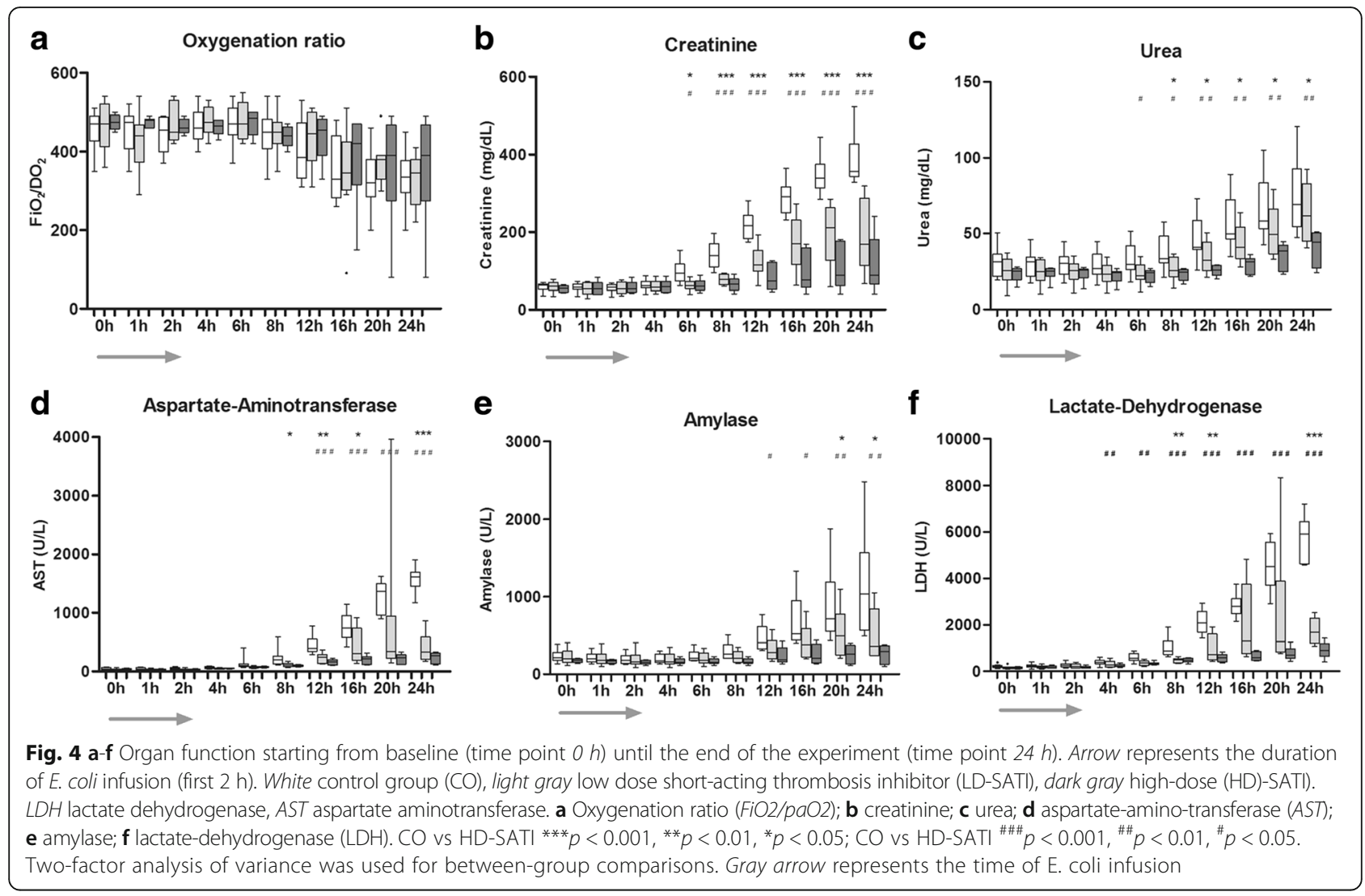

Temperature was not different between the groups (data not shown). Similarly, circulating LBP concentrations were not different between the groups over the observation period.

\section{Hemodynamic response to sepsis}

MAP, CI, SVRI and PVRI significantly changed over time (all $p<0.03$ ) (Table 1). Administration of SATI led to higher $\mathrm{CI}$, and lower SVRI and PVRI. Base excess
(BE) significantly decreased over the observation period in all groups $(p<0.0001)$. However, SATI administration resulted in significantly improved $\mathrm{BE}$ in both treatment arms compared to the CO group from TP 8 until the end of the experiment $(p<0.01)$. Noradrenaline was required to maintain MAP $>70 \mathrm{mmHg}$ in seven out of eight animals in the CO group, compared to three out of eight in the LD-SATI group and one out of six in the HD-SATI group $(p<0.03)$. Nevertheless, two baboons

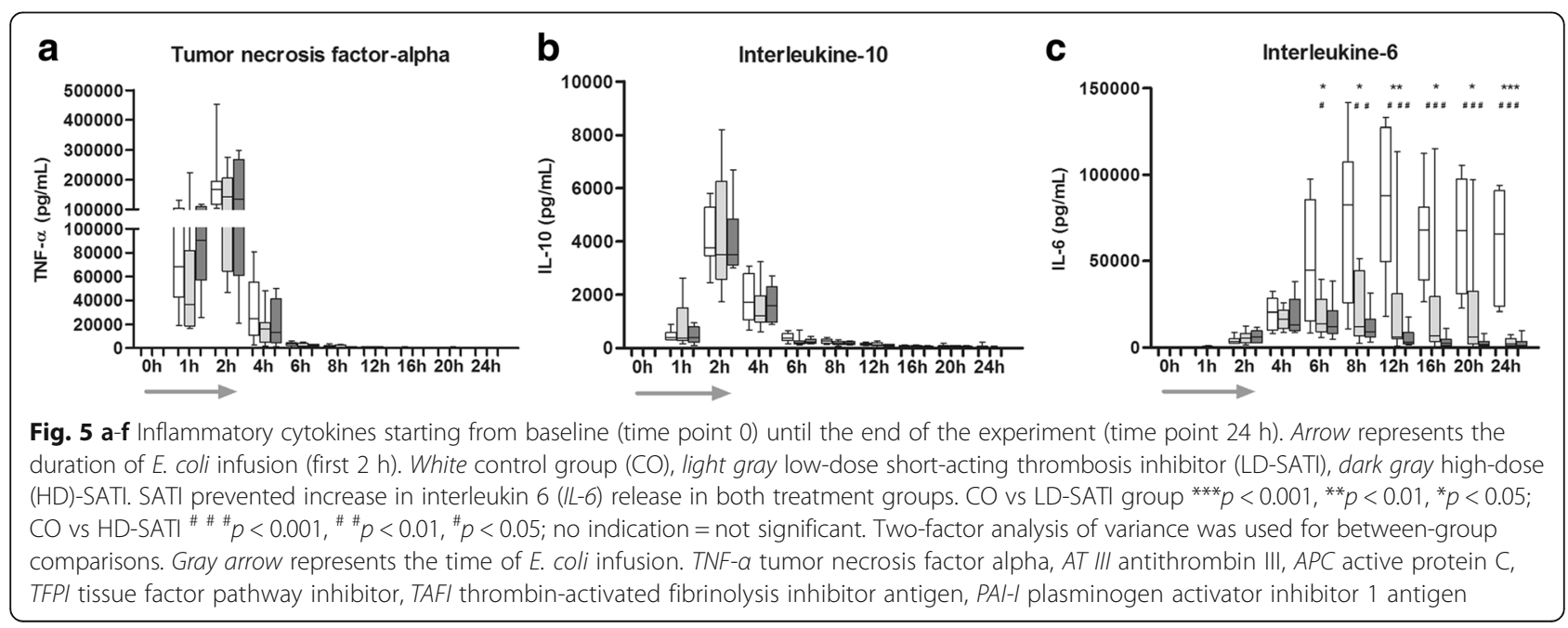




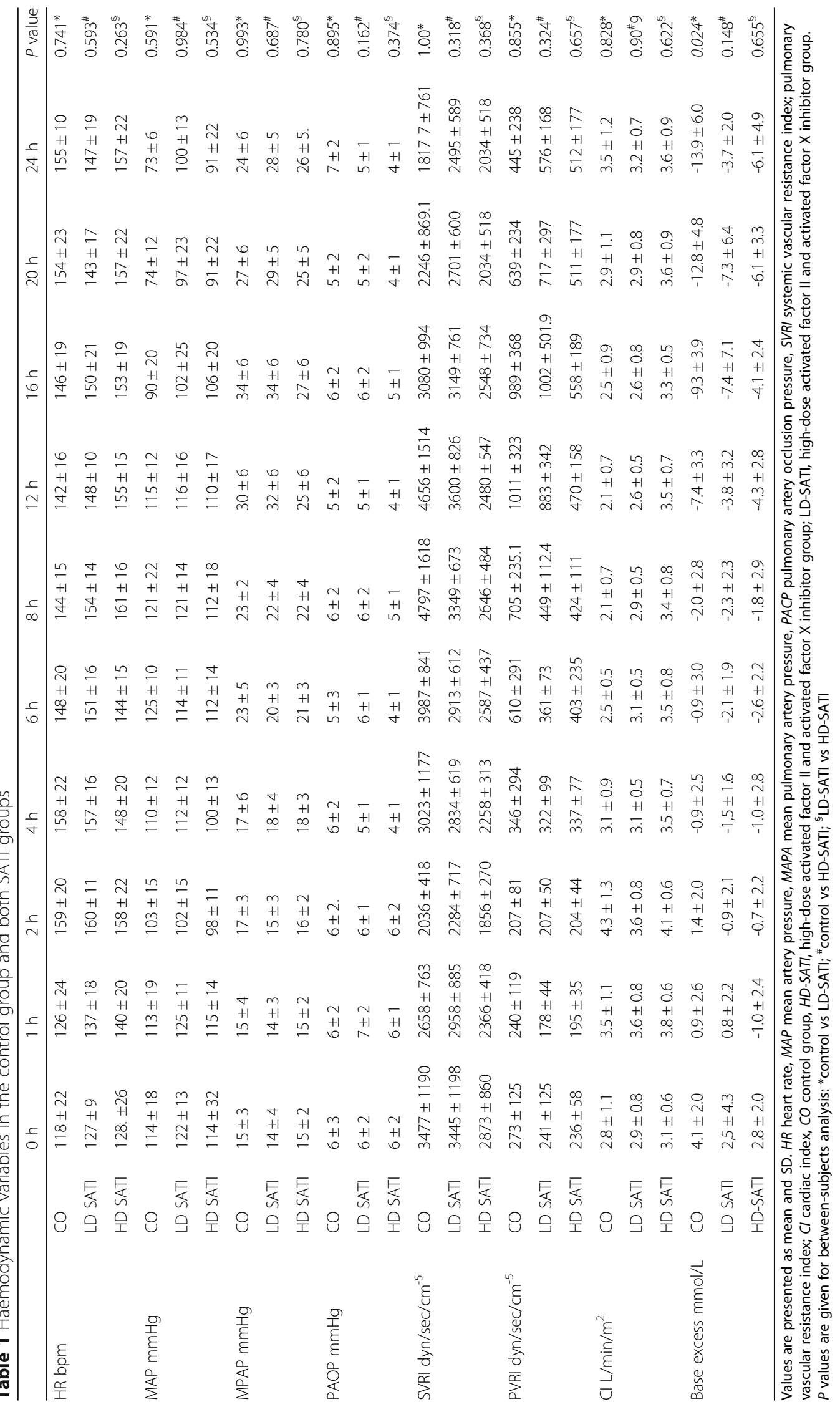


died in the LD-SATI group: one after $16 \mathrm{~h}$ and one after $19 \mathrm{~h}$ due to hemodynamic failure but not to bleeding complications.

\section{Blood cell count after E. coli infusion}

All animals developed anemia and neutropenia after infusion of E. coli (Table 2). No significant differences were observed between the $\mathrm{CO}$ and the SATI groups.

\section{Discussion}

This is the first study investigating the effect of dual inhibition of thrombin and activated FX in a non-human primate model of severe Gram-negative sepsis. Posttreatment with SATI starting $2 \mathrm{~h}$ and 15 minutes after the initiation of sepsis blocked thrombin and factor Xmediated activation of coagulation and maintained natural inhibitors such as ATIII and APC. Moreover, profibrinolytic pathways were preserved in particular in those animals that received HD-SATI. Most importantly, treatment with SATI, regardless of the dose, strongly attenuated sepsisinduced organ injury reflected by the reduced release of organ-specific enzymes and even prevented anuria, which developed after $8 \mathrm{~h}$. The results of the current study indicate that dual inhibition of thrombin and FXa by SATI is capable of attenuating the severity of DIC and counteracts sepsis-induced organ dysfunction. Furthermore, SATI abolished the late inflammatory response as indicated by diminished release of circulating IL-6.

We used a baboon model, which closely recapitulates early events in human sepsis such as hyperdynamic response in the acute phase, even with adequate fluid resuscitation [24-26]. The bacterial challenge was severe enough to provoke a massive inflammatory response, strong activation of coagulation and sustained organ damage, but allowed the majority of animals to survive for $24 \mathrm{~h}$; only two baboons died before completion of the experiment. At the end of the bacterial infusion, all groups had a similar septic phenotype: high inflammatory response, hemodynamic instability and strong upregulation of procoagulant pathways.

The protracted infusion of E.coli strongly increased thrombin generation as indicated by a very high concentration of TAT complexes in the CO group. ATIII binds thrombin in a 1:1 ratio leading to formation of TAT [27]. In contrast, SATI treatment robustly reduced active thrombin binding to ATIII, resulting in lower TAT via two modalities. On one hand SATI blocks the active site of thrombin and consequently inhibits binding to ATIII. On the other hand it can be partly related to the reduced thrombin production by SATI via blockade of Xa activity (dual-action SATI concept). Thus, ATIII decreased significantly more in the CO group compared to both SATI groups due to higher consumption by free thrombin, consequently leading to stronger elevation in TAT in the
$\mathrm{CO}$ group. Other inhibitors such as APC and TFPI increased to a greater extent in the $\mathrm{CO}$ group compared to the SATI groups up to the end of the experiment. As mentioned, this effect could be explained by the fact that SATI blocks the active thrombin site, consequently hindering binding on TM. Thus, activation of protein $C$ is downregulated by SATI, which led to a higher concentration of APC in the $\mathrm{CO}$ group.

In the present study, the marked increase in PAI-1 was significantly ameliorated by HD-SATI. High concentrations of inflammatory cytokines such as TNF- $\alpha$ and IL-1 provoke release of PAI-1 into the bloodstream, causing sustained inhibition of thrombolysis [28]. In experimental and clinical studies, high PAI-1 has been strongly associated with poor outcome in severe sepsis $[29,30]$.

Additionally, we observed strong upregulation of TAFI in the $\mathrm{CO}$ group, which further hinders lysis. This effect was in part counteracted by high APC in the CO group, which in turn inactivates PAI-1. D-dimer elevation in the $\mathrm{CO}$ group could result from a sustained increase in fibrin formation (related to high thrombin availability) rather than being per se a marker of upregulated lysis.

Interestingly thrombocytopenia was not different between the groups. This is a remarkable finding in the light that SATI robustly abolished thrombin generation as indicated by significantly lower TAT. A possible explanation for this observation is that this severe life-threatening sepsis resulted in sustained upregulation of platelet action, which in turn caused pronounced thrombocytopenia independent of therapeutic intervention.

Hemodynamic instability and organ dysfunction is a common issue in severe sepsis and septic shock [10]. Intravascular fibrin formation results in limited nutrient delivery to vital organs and consecutive end-organ damage. In particular, tissues with high oxygen demand such as the kidneys are susceptible to compromised end-organ perfusion [31]. The BE was significantly lower in the $\mathrm{CO}$ group compared to both SATI treatment arms, which might suggest improved microvascular blood flow. While all $\mathrm{CO}$ animals stopped urine production after the first $8 \mathrm{~h}$, remarkably, diuresis in both SATI groups was maintained until the end of the experiment. The sepsis-induced organ dysfunction in our study closely meets the most recent definition of sepsis [32]. Over the study course, the lung, liver, kidney and pancreas function deteriorated. The life-threatening organ failure observed in the $\mathrm{CO}$ group was significantly improved in baboons treated with SATI. In particular, HD-SATI preserved organ function. It is important to note that, in both SATI groups compared to the $\mathrm{CO}$, fewer catecholamines were necessary to maintain MAP $>70 \mathrm{mmHg}$.

Several randomized controlled studies have been conducted using natural anticoagulants such as ATIII [13, 14], recombinant human APC $[12,16]$, recombinant human 


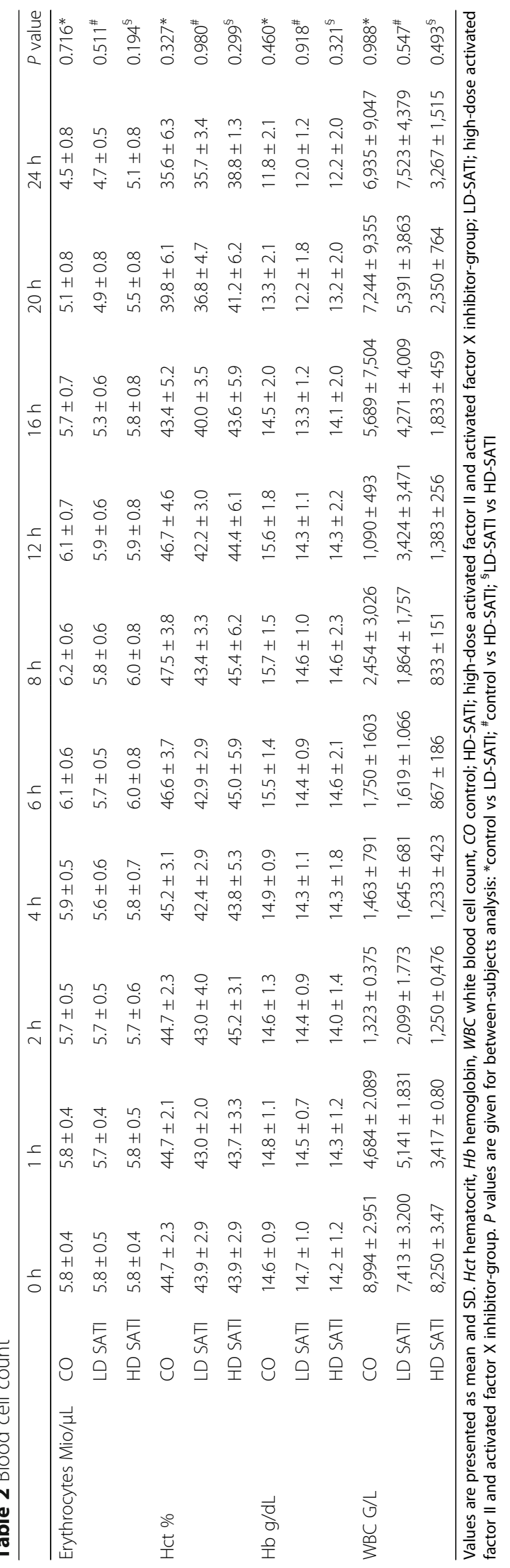


TFPI [12, 15] or recombinant human TM [33] in patients with sepsis. Those drugs reduced the hemostatic abnormalities in sepsis but did not improve survival [34]. However, on post-hoc subgroup analysis restricted to those patients with overt DIC, there were survival benefits in both the Kybersept study and the PROWESS trial $[17,18]$. This strongly implies the need for a more homogenous stratification of patients with sepsis into those with overt DIC and those with sepsis but non-overt DIC. Patients with established DIC could benefit from treatment with anticoagulants such as ATIII or APC. Our data provide evidence that dual inhibition of both thrombin and FXa robustly ameliorates organ dysfunction, most likely by improving microvascular nutrient blood flow based on diminished intravascular fibrin formation.

Another important finding of the current study is that SATI treatment was highly effective in preventing the progression of DIC but there were no signs that it provoked any severe bleeding events, even in the HD-SATI group. This is in strong contrast to studies testing therapeutic administration of ATIII and APC, which led to life-threatening bleeding complications [12, 13]. Giardino et al. confirmed that compared to selective inhibition alone, dual inhibition of thrombin and FXa exerts a superior antithrombotic efficacy with a tendency toward diminished bleeding [22].

The bacterial infusion provoked a potent release of initial inflammatory cytokines prior to the onset of treatment. Thus, SATI could not have meaningful effect on the time course and magnitude of TNF- $\alpha$ and IL-10, which peaked at the end of the bacterial challenge, long before the start of treatment. IL-6 release occurs later than that of TNF- $\alpha$ and IL-10; however, the pattern of production is somewhat different in various models [35]. Both SATI groups were effective in attenuating late IL-6 release in the bloodstream. IL- 6 has been shown to be, at least in part, under the control of the initial TNF- $\alpha$ release [36]. Considering that TNF- $\alpha$ release prior to the SATI treatment was not affected, we assume that SATI per se has a direct effect on IL-6 neo-synthesis. Alternatively, the observed effect could be a consequence of diminished availability of thrombin, which has strong pro-inflammatory properties [4].

\section{Limitation}

Due to the limited observation period, our study does not provide any relevant outcome information on the efficacy of SATI at later stages. Early empiric antibiotic treatment is an essential part of initial treatment of patients with sepsis [10]. In the current short-term study, we did not use any additional antibiotic therapy, which could potentially have influence on the time course of sepsis-related organ damage. However, SATI treatment according to our protocol was effective in improving sepsis-related organ injury.

The duration of the study was too short to completely rule out substantial bleeding complications at a later stage. However, even in the HD-SATI group relevant bleeding episodes were not observed, including external bleeding and mucosa or gastrointestinal bleeding, which would have been apparent after harvesting the organs post mortem. Two animals in the LD-SATI group died due to hemodynamic decompensation. However, it has to be noted that the study was not powered to show any survival differences between groups.

\section{Conclusion}

SATI treatment diminished thrombin formation and preserved anticoagulant and fibrinolytic pathways. This effect coincided with an effective and robust reduction in severe sepsis-induced organ injury in both SATI treatment groups. Moreover, dual inhibition of thrombin and aFX with SATI strongly ameliorated late inflammation in baboons with sepsis.

\section{Key messages}

- Bacterial invasion prompts a compelling upregulation of the coagulation system and inhibits anticoagulant and fibrinolytic pathways, which results in widespread microvascular fibrin deposition

- Dual inhibition of activated FII and activated FX (SATI) diminishes thrombin formation and preserves anticoagulant and fibrinolytic pathways

- SATI administration strongly ameliorates IL-6 release in severe sepsis

- SATI robustly attenuates sepsis-induced organ damage and protects organ function

\section{Abbreviations}

ALT: Alanine-aminotransferase; APC: Activated protein C; AST: Aspartateaminotransferase; AT III: Antithrombin III; BE: Base excess; BW: Body weight; Cl: Cardiac index; CK: Creatine kinase; CO: Control group; CVP: Central venous pressure; DIC: Disseminated intravascular coagulation; ELISA: Enzyme-linked immunosorbent assay; $\mathrm{FiO}_{2} / \mathrm{paO}_{2}$ : Oxygenation ratio; FIX: Factor IX;

FVII: Factor VII; FX: Factor X; Hb: Hemoglobin; Hct: Hematocrit; HD-SATI: High dose short acting thrombosis inhibitor group; IL-10: Interleukin-10;

IL-6: Interleukin-6; LBP: Lipopolysaccharide binding protein; LDH: Lactatedehydrogenase; LD-SATI: Low dose short acting thrombosis inhibitor group; MAP: Mean arterial pressure; PAl-1: Plasminogen activator inhibitor 1 antigen; PAOP: Pulmonary artery occlusion pressure; PVRI: Pulmonary vascular resistance index; SATI: Short-acting thrombosis inhibitor; SVRI: Systemic vascular resistance index; TAFI: Thrombin activated fibrinolysis inhibitor antigen; TAT: Thrombin-antithrombin-complexes; TF: Tissue factor; TFPI: Tissue factor pathway inhibitor; TM: Thrombomodulin; TNF-a: Tumor necrosis factor-a; TP: Time point

\section{Acknowledgements}

The authors gratefully thank Francoise DeWet, and Zafar Khakpour for excellent technical assistance during the experiments. Moreover, we want to thank Anna Khadem, Claudia Grießbach and Mohammad Jafarmadar for blood sample analyses. The authors highly appreciate the critical review of the manuscript 
and valuable comments of Dr Marcin Osuchowski for substantially contributing to the quality of the paper.

\section{Funding}

The study was supported by an unrestricted grant from Bayer Healthcare, Wuppertal.

\section{Availability of data and materials}

The data generated or analyzed during this study are available from the corresponding author on reasonable request.

\section{Authors' contributions}

$H S, H R, V L, S H, S B$ and MvG designed the experiment. MvG, HS, SH, JR and $V L$ performed the experiments. UK performed statistical analysis. HS, MvG and $\mathrm{SH}$ wrote the manuscript. SB, JR, UK and HR revised the manuscript. All authors read and approved the final manuscript.

\section{Competing interests}

$\mathrm{HS}$ received honorarium for participation in advisory board for Bayer Healthcare. SH and VL are employees of Bayer Healthcare. SB, HR, JR and MvG declare no conflict of interest.

\section{Consent for publication}

Not applicable.

\section{Ethics approval}

The study was approved by the Institutional Animal Care use Committee at Free State University Bloemfontein, South Africa (Number: 03/2010).

\section{Author details}

${ }^{1}$ Ludwig Boltzmann Institute for Experimental and Clinical Traumatology, AUVA Research Centre, Vienna, Austria. ${ }^{2}$ Department of Anesthesiology and Intensive Care Medicine, AUVA Trauma Centre Salzburg, Academic Teaching Hospital of the Paracelsus Medical University, Dr. Franz Rehrl Platz 5, 5020 Salzburg, Austria. ${ }^{3}$ Department of Experimental Trauma Surgery, Trauma Surgery, Klinikum rechts der Isar, Technical University of Munich, Munich, Germany. ${ }^{4}$ Bayer Pharma AG, Acute Care Research, Wuppertal, Germany. ${ }^{5}$ TT SPSS Statistics, Salzburg, Austria. ${ }^{6}$ Department of Haematology and Cell Biology, Faculty of Health Sciences, University of the Free State, Bloemfontein, Free State, South Africa.

Received: 3 November 2016 Accepted: 15 February 2017

Published online: 13 March 2017

\section{References}

1. Angus DC, Linde-Zwirble WT, Lidicker J, Clermont G, Carcillo J, Pinsky MR. Epidemiology of severe sepsis in the United States: analysis of incidence, outcome, and associated costs of care. Crit Care Med. 2001;29:1303-10.

2. Kumar G, Kumar N, Taneja A, Kaleekal T, Tarima S, McGinley E, et al. Milwaukee Initiative in Critical Care Outcomes Research Group of Investigators. Nationwide trends of severe sepsis in the 21st century (2000-2007). Chest. 2011;140:1223-31.

3. Esmon CT. The interactions between inflammation and coagulation. $\mathrm{Br}$ Haematol. 2005:131:417-30.

4. Angus DC, van der Poll T. Severe sepsis and septic shock. New Engl J Med 2013:29(369):840-51.

5. Levi M, van der Poll T. Inflammation and coagulation. Crit Care Med. 2010;38(2 Suppl):S26-34.

6. Allen KS, Sawheny E, Kinasewith GT. Anticoagulant modulation of inflammation in severe sepsis. World J Crit Care Med. 2015;4:105-15.

7. Fourrier F. Severe sepsis, coagulation, and fibrinolysis: dead end or one way? Crit Care Med. 2012;40:2704-8

8. Levi M, Ten Cate H. Disseminated intravascular coagulation. N Engl J Med. 1999;341:586-92.

9. van der Poll T, de Jonge E, Levi M. Regulatory role of cytokines in disseminated intravascular coagulation. Semin Thromb Hemost. 2001;27:639-51.

10. Dellinger RP, Levy MM, Rhodes A, Annane D, Gerlach H, Opal SM, et al. Surviving Sepsis Campaign Guidelines Committee including the Pediatric Subgroup: international guidelines for management of severe sepsis and septic shock: 2012. Crit Care Med. 2013;41:580-637.
11. Dhainaut JF, Yan SB, Joyce DE, Pettilä V, Basson B, Brandt JT, et al. Treatment effects of drotrecogin alfa (activated) in patients with severe sepsis with or without overt disseminated intravascular coagulation. J Thromb Haemost. 2004:2:1924-33.

12. Simon S, Light B, Spapen H, Stone J, Seibert A, Peckelsen C, et al. Efficacy and safety of tifacogin (recombinant tissue factor pathway inhibitor) in severe sepsis: a randomized controlled trial. JAMA. 2003;290:238-47.

13. Eid A, Wiedermann CJ, Kinasewitz GT. Early administration of high-dose antithrombin in severe sepsis: single center results from the KyberSept-trial. Anesth Analg. 2008;107:1633-8.

14. Warren BL, Eid A, Singer P, Pillay SS, Carl P, Novak I, et al. Caring for the critically ill patient. High-dose antithrombin III in severe sepsis: a randomized controlled trial. JAMA. 2001;286:1869-78.

15. Wunderink RG, Laterre PF, Francois B, Perrotin D, Artigas A, Vidal LO, et al. Recombinant tissue factor pathway inhibitor in severe community-acquired pneumonia: a randomized trial. Am J Respir Crit Care Med. 2011;183:1561-8.

16. Ranieri VM, Thompson BT, Barie PS, Dhainaut JF, Douglas IS, Finfer S, et al. Drotrecogin alfa (activated) in adults with septic shock. N Engl J Med. 2012;366:2055-64

17. Kienast J, Juers M, Wiedermann CJ, Hoffmann JN, Ostermann H, Strauss R, et al. Treatment effects of high-dose antithrombin without concomitant heparin in patients with severe sepsis with or without disseminated intravascular coagulation. J Thromb Haemost. 2006;4:90-7.

18. Bernard GR, Vincent JL, Laterre PF, LaRosa SP, Dhainaut JF, Lopez-Rodriguez A, et al. Efficacy and safety of recombinant human activated protein $C$ for severe sepsis. New Engl J Med. 2001;344:699-709.

19. Iba T, Saitoh D, Wada H, Asakura H. Efficacy and bleeding risk of antithrombin supplementation in septic disseminated intravascular coagulation: a secondary survey. Crit Care. 2014;18:497.

20. Pernerstorfer T, Hollenstein U, Hansen JB, Stohlawetz P, Eichler HG, Handler S, et al. Lepirudin blunts endotoxin-induced coagulation activation. Blood. 2000:95:1729-34

21. Hollenstein UM, Pernerstorfer $T$, Homoncik M, Hansen JB, Finzen $H$, Handler $S$, Jilma B. Effect of factor $X$ inhibition on coagulation activation and cytokine induction in human systemic inflammation. J Infect Dis. 2002;186:1270-6.

22. Giardino EC, Haertlein BJ, de Garavilla L, Costanzo MJ, Damiano BP, Andrade-Gordon P, Maryanoff BE. Cooperative antithrombotic effect from the simultaneous inhibition of thrombin and factor Xa. Blood Coagul Fibrinolysis. 2010;21:128-34.

23. Agnelli G, Gallus A, Goldhaber SZ, Haas S, Huisman MV, Hull RD, et al. Treatment of proximal deep-vein thrombosis with the oral direct factor $\mathrm{Xa}$ inhibitor rivaroxaban (BAY 59-7939): the ODIXa-DVT (Oral direct factor Xa inhibitor BAY 59-7939 in patients with acute symptomatic deep-vein thrombosis) study. Circulation. 2007;116:180-7.

24. Redl HR, Martin U, Khadem A, Pelinka LE, van Griensven M. Anti-L-selectin antibody therapy does not worsen the post septic course in a baboon model. Crit Care. 2005;9:R735.

25. Schlag G, Redl H, Davies J. Live Escherichia coli sepsis models in baboons. In: Schlag G, Redl H, editors. Pathophysiology of shock, sepsis, and organ failure. Berlin, Heidelberg: Springer; 1993. p. 1076-107.

26. Schlag G, Redl H, Hallström S, Radmore K, Davies J. Hyperdynamic sepsis in baboons: I. Aspects of hemodynamics. Circ Shock. 1991;34:311-8.

27. Fourrier F. Clinical trial results with antithrombin III in sepsis. Crit Care Med. 2000;28:S38-43.

28. Martin NB, Jamieson A, Tuffin DP. The effect of interleukin-4 on tumour necrosis factor-alpha induced expression of tissue factor and plasminogen activator inhibitor-1 in human umbilical vein endothelial cells. Thromb Haemost. 1993;70:1037-42

29. Raeven P, Drechsler S, Weixelbaumer KM, Bastelica D, Peiretti F, Klotz A, et al. Systemic inhibition and liver-specific over-expression of PAI-1 failed to improve survival in all-inclusive populations or homogenous cohorts of CLP mice. J Thromb Haemost. 2014;12:958-69.

30. Lorente L, Martín MM, Borreguero-León JM, Solé-Violán J, Ferreres J, Labarta L, Diaz C, et al. Sustained high plasma plasminogen activator inhibitor-1 levels are associated with severity and mortality in septic patients. Thromb Res. 2014:134:182-6.

31. Benes J, Chvojka J, Sykora R, Radej J, Krouzecky A, Novak I, Matejovic M. Searching for mechanisms that matter in early septic acute kidney injury: an experimental study. Crit Care. 2011;15:R256.

32. Seymour CW, Rosengart MR. Septic Shock. Advances in diagnosis and treatment. JAMA. 2015;18(314):708-17. 
33. Saito H, Maruyama I, Shimazaki S, Yamamoto Y, Aikawa N, Ohno R, et al. Efficacy and safety of recombinant human soluble thrombomodulin (ART-123) in disseminated intravascular coagulation: results of a phase III, randomized, double-blind clinical trial. J Thromb Haemost. 2007:5:31-41.

34. Wada H, Matsumoto T, Yamashita Y, Hatada T. Disseminated intravascular coagulation: testing and diagnosis. Clin Chim Acta. 2014;436:130-4.

35. Redl H, Schlag G, Bahrami S, Schade U, Ceska M, Stütz P. Plasma neutrophilactivating peptide-1/interleukin-8 and neutrophil elastase in a primate bacteremia model. J Infect Dis. 1991;164:383-8.

36. Waage A, Redl H, Schlag G, Schade U. The cytokine network in sepsis II: IL-1 and IL-6. In: Schlag G, Redl H, editors. Pathophysiology of shock, sepsis, and organ failure. Berlin, Heidelberg: Springer; 1993. p. 491-8.

Submit your next manuscript to BioMed Central and we will help you at every step:

- We accept pre-submission inquiries

- Our selector tool helps you to find the most relevant journal

- We provide round the clock customer support

- Convenient online submission

- Thorough peer review

- Inclusion in PubMed and all major indexing services

- Maximum visibility for your research

Submit your manuscript at www.biomedcentral.com/submit 